\title{
Late Subaxial Lesion after Overcorrected Occipitocervical Reconstruction in Patients with Rheumatoid Arthritis
}

\author{
Akira Iwata ${ }^{1}$, Kuniyoshi Abumi ${ }^{2}$, Masahiko Takahata ${ }^{1}$, Hideki Sudo $^{3}$, \\ Katsuhisa Yamada ${ }^{1}$, Tsutomu Endo ${ }^{1}$, Norimasa Iwasaki ${ }^{1}$ \\ ${ }^{I}$ Department of Orthopaedic Surgery, Faculty of Medicine and Graduate School of Medicine, Hokkaido University, Sapporo, Japan \\ ${ }^{2}$ Sapporo Orthopaedic Hospital-Center for Spinal Disorders, Sapporo, Japan \\ ${ }^{3}$ Department of Advanced Medicine for Spine and Spinal Cord Disorders, Hokkaido University Graduate School of Medicine, Sapporo, Japan
}

Study Design: Retrospective case-control study, level 4.

Purpose: To clarify the risk factors for late subaxial lesion after occipitocervical (O-C) reconstruction. We examined cases requiring fusion-segment-extended (FE) reconstruction in addition to/after 0-C reconstruction.

Overview of Literature: Patients with rheumatoid arthritis (RA) frequently require 0-C reconstruction surgery for cranio-cervical lesions. Acceptable outcomes are achieved via indirect decompression using cervical pedicle screws and occipital plate-rod systems. However, late subaxial lesions may develop occasionally following 0-C reconstruction.

Methods: 0-C reconstruction using cervical pedicle screws and occipital plate-rod systems was performed between 1994 and 2007 in 113 patients with RA. Occipito-atlanto-axial (0-C2) reconstruction was performed for 89 patients, and occipito-subaxial cervical (0-under C2) reconstruction was performed for 24 patients. We reviewed the cases of patients requiring FE reconstruction (fusion extended group, FEG) and 26 consecutive patients who did not require FE reconstruction after a follow-up of $>5$ years (non-fusion extended group, NEG) as controls.

Results: FE reconstructions were performed for nine patients at an average of 45 months (range, 24-180 months) after 0-C reconstruction. Of the 89 patients, three (3\%) underwent FE reconstruction in cases of 0-C2 reconstruction. Of the 24 patients, five (21\%) underwent FE reconstruction in cases of 0 -under $\mathrm{C} 2$ reconstruction ( $p=0.003$, Fisher exact test). Age, sex, RA type, and neurological impairment stage were not significantly different between FEG and NEG. 0-under C2 reconstruction, larger correction angle $\left(4^{\circ}\right.$ per number of unfixed segment), and $\mathrm{O}-\mathrm{C} 7$ angle change after $\mathrm{O}-\mathrm{C}$ reconstruction were the risk factors for late subaxial lesions on radiographic assessment.

Conclusions: Overcorrection of angle at fusion segments requiring 0-C7 angle change was a risk factor for late subaxial lesion in patients with RA with fragile bones and joints. Correction should be limited, considering the residual mobility of the cervical unfixed segments.

Keywords: Reconstruction; Pedicle screw; Rheumatoid arthritis; Atlanto occipital joint

Received Mar 22, 2018; Revised Sep 4, 2018; Accepted Sep 27, 2018

Corresponding author: Akira Iwata

Department of Orthopaedic Surgery, Faculty of Medicine and Graduate School of Medicine, Hokkaido University, North-15, West-7, Kita-ku, Sapporo, Hokkaido 060-8638, Japan

Tel: +81-11-706-5934, Fax +81-11-706-6054, E-mail: iwataakira0126@yahoo.co.jp 


\section{Introduction}

Patients with rheumatoid arthritis (RA) often exhibit neurological deficits in terms of atlanto-axial and vertical subluxations [1], which may deteriorate sudden death $[2,3]$. Sudden death can be caused by direct compression of the brain stem or ischemia that is secondary to the compression of vertebral arteries, anterior spinal arteries, and small perforating arteries of the brain stem and the spinal cord. These result from the destruction of the transverse ligament, alar ligament, lateral masses of the atlas, and basilar processes of the skull and bone resorption in the occipital condyles [4]. Advances in surgical technique have enabled the recovery of neurological deficits via surgical intervention $[5,6]$, thereby improving the prognosis of patients with RA $[7,8]$.

Indirect anterior spinal cord decompression technique via occipitocervical reconstruction ( $\mathrm{O}-\mathrm{C}$ reconstruction)

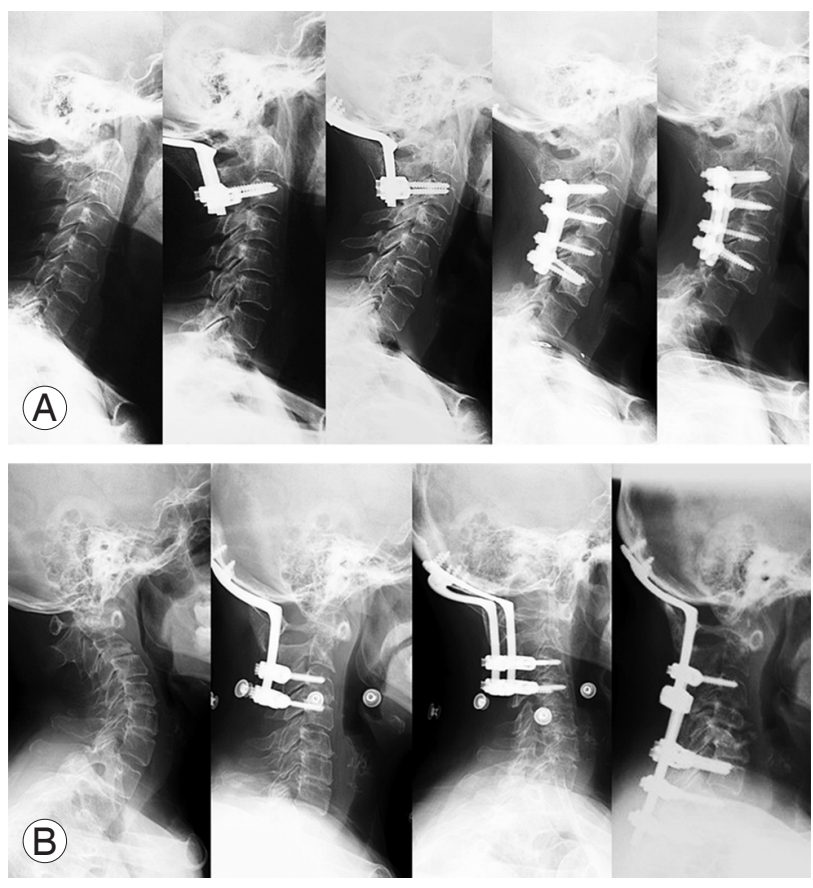

Fig. 1. The case of the patient who required fusion extension reconstruction after 0 -C reconstruction. (A) A 63-year-old woman with rheumatoid arthritis underwent $0-\mathrm{C} 3$ reconstruction. Forty-three months thereafter, she underwent reconstruction of fusion segment extension to $\mathrm{C} 6$ by subluxation at inferior segment of $\mathrm{C} 4 / 5$. Forty-five months later, she developed subluxation again at inferior adjusted fusion segment of $\mathrm{C} 6 / 7$, and she took again the reconstruction of fusion segment extension to T3. (B) A 63-year-old woman with rheumatoid arthritis underwent $0-\mathrm{C} 4$ reconstruction. Fifty-seven months thereafter, she underwent fusion extension reconstruction to T2 for the late C5 vertebral fracture with myelopathy. $0-C$, occipitocervical. using cervical pedicle screw aimed to normalize basilar invagination and forward subluxation of the atlas on the axis [9], thus resolving subarachnoid space encroachment and preventing progression. Acceptable outcomes were achieved by the current indirect posterior reconstruction. However, several problems of indirect posterior reconstruction emerged with an increase in the $\mathrm{O}-\mathrm{C}$ reconstruction cases. Acquiring anchor at axis by high-riding vertebral artery is difficult, particularly in patients with RA who require surgical intervention [10-12]. Dyspnea and dysphagia occur with more flexion position at $>10^{\circ}$ before surgery because of the reduction in the oropharyngeal cross-sectional area [13]. We have experienced some cases with late subaxial lesion after $\mathrm{O}-\mathrm{C}$ reconstruction that required fusion-segment-extended reconstruction (FE reconstruction) (Fig. 1). The etiology and risk factors for late subaxial lesion after $\mathrm{O}-\mathrm{C}$ reconstruction have not been studied thus far.

In this study, we aimed to clarify the risk factors for late subaxial lesion after $\mathrm{O}-\mathrm{C}$ reconstruction in patients with $\mathrm{RA}$. We examined cases requiring $\mathrm{FE}$ reconstruction on radiographic assessment.

\section{Materials and Methods}

This study was approved by the Institutional Review Board (IRB) of Hokkaido University (IRB approval no., 011-0338) and has been performed as per the ethical standards of the 1964 Declaration of Helsinki and its later amendments or comparable ethical standards. Informed consent was not obtained from the participants because of the IRB provision for the retrospective-nature of the study. On behalf of all the authors, the corresponding author states that there is no conflict of interest with respect to this study. This was a retrospective case-control study, level 4 .

Of the 113 patients with RA who underwent $\mathrm{O}-\mathrm{C}$ reconstruction using indirect decompression technique during 1993-2007 without tight control with biological drugs, 89 and 24 patients underwent occipito-atlanto-axial (O-C2) and occipito-subaxial cervical (O-under $\mathrm{C} 2$ ) reconstructions, respectively. The indications to extend under $\mathrm{C} 2$ were the presence of subaxial lesions below $\mathrm{C} 2 / 3$ or difficulty in inserting $\mathrm{C} 2$ pedicle screws by the high-riding vertebral artery. The lowest instrumented vertebra was considered as the vertebra below subaxial lesions without extremely small pedicle, anomalies of the vertebral artery, and extremely 
large angle of the pedicle axis to the sagittal plane.

FE reconstructions were required 9 times in seven patients. This study enrolled seven patients with FE reconstruction (fusion extended group, FEG) conducted at an average of 45 months (range, 24-180 months), and 26 consecutive patients obtained radiograph without FE reconstruction after $\mathrm{O}-\mathrm{C}$ reconstruction for $>5$ years (non-fusion extended group, NEG) from $113 \mathrm{O}-\mathrm{C}$ reconstructions as a control group. The patients who required instrumentation removal because of infection and those with fusion from the occipital bone to the thoracic vertebra were excluded from the analyses.

Background demographic data regarding age, sex, type of RA (subsets with least erosive disease, more erosive disease, and mutilating disease), Ranawat classification of neurological impairment, number of fusion segments at $\mathrm{O}-\mathrm{C}$ reconstruction, period from $\mathrm{O}-\mathrm{C}$ reconstruction to FE reconstruction, and pathogenesis requiring FE reconstruction were collected.

Radiographic assessments were conducted using the neutral position of the lateral cervical spine radiographs before and after the surgery; these were obtained at $>1.5$ $\mathrm{m}$ away from the cassette, with no magnification in the standing position as possible while keeping the eyes forward (horizontal). Measurements were the extent of basilar invagination (Ranawat value), extent of forward subluxation of the atlas on the axis, angle from the occipital bone to the caudal fixed vertebra (O-CLFV angle), and the occipital bone to the 7th cervical vertebra $(\mathrm{O}-\mathrm{C} 7$ angle). The change in the O-CLFV angle after the reconstruction surgery was determined as the correction angle. The differences between FEG and NEG were examined on radiographic assessment.

Statistical analyses were performed using JMP Pro ver. 12 (SAS Institute Inc., Cary, NC, USA). Fisher's exact test was performed for comparing categorical data. $T$-test was conducted for assessing continuous variables. Data are expressed as mean and $95 \%$ confident interval for continuous values. The level of significance was defined as $p<0.05$. The optimal cut-off values were examined using receiver operating characteristic (ROC) curve.

\section{Results}

\section{Demographic data of patients requiring fusion-seg- ment-extended reconstruction}

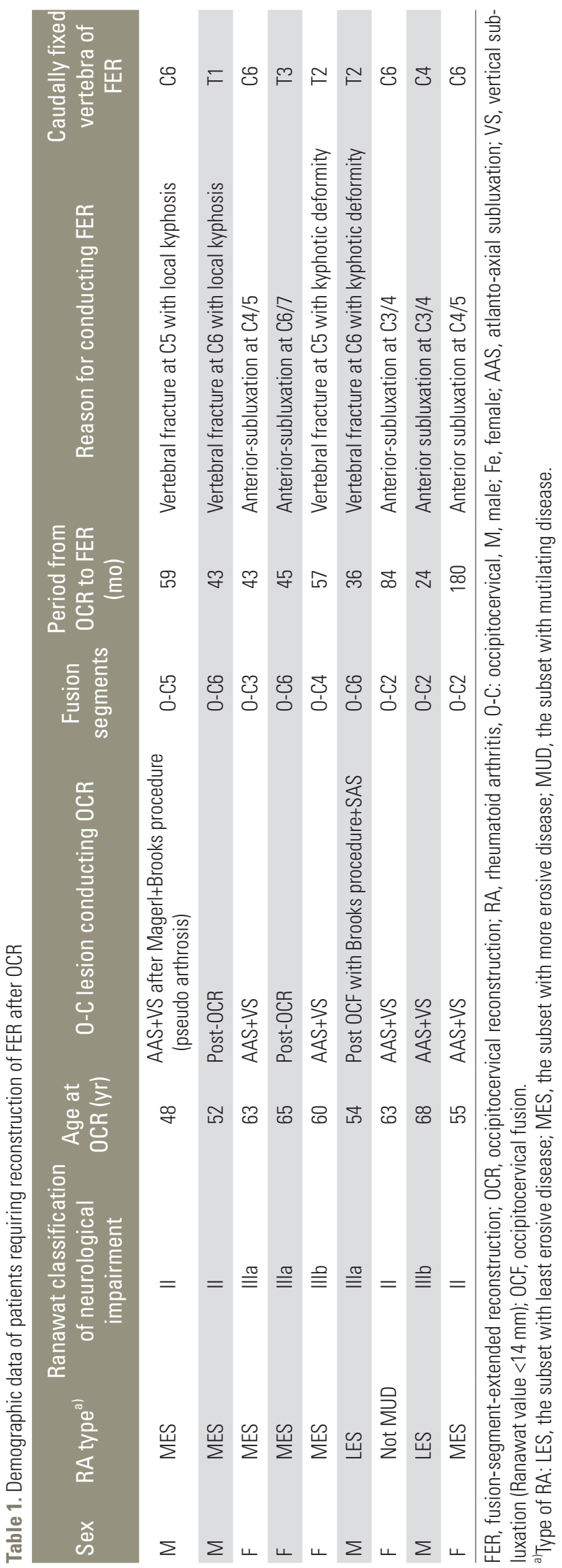


In the nine instances in seven patients, $\mathrm{FE}$ reconstructions were required after $\mathrm{O}-\mathrm{C}$ reconstruction. Four patients had a vertebral fracture at the caudal fixed vertebra and five had subluxation at the end of the fixed segment. Vertebral fractures or subluxations cause local kyphotic deformity. $\mathrm{FE}$ reconstruction was performed at an average of 45 months (range, 24-180 months) after O-C reconstruction (Table 1).

\section{Background data in the non-fusion extended group and the fusion extended group}

There were no significant differences in the background demographic data before $\mathrm{O}-\mathrm{C}$ reconstruction, age at $\mathrm{O}-\mathrm{C}$ reconstruction, sex, Ranawat classification of neurological impairment, type of RA, extent of vertical subluxation (Ranawat value), extent of forward subluxation of the atlas on the axis (space available for the spinal cord and atlanto-dental interval), O-CLFV angle, and O-C7 angle of the two groups (Table 2).

\section{Effect of fusion segments}

In cases of $\mathrm{O}-\mathrm{C} 2$ reconstruction, only $3 \%$ (three of 89 patients) required FE reconstruction; however, 25\% (six of 24 patients) required $\mathrm{FE}$ reconstruction in cases of $\mathrm{O}$ under $\mathrm{C} 2$ reconstruction $(p<0.001$, Fisher exact test).

\section{Change of angulation and translation in the non- fusion extended group and the fusion extended group}

The correction angle was $9.9^{\circ} \pm 4.7^{\circ}$ and $3.4^{\circ} \pm 2.2^{\circ}$ in the FEG and NEG, respectively ( $p=0.217, t$-test). The correction angle per number of unfixed segment was $9.6^{\circ} \pm 2.0^{\circ}$ and $0.8^{\circ} \pm 1.0^{\circ}$ in the FEG and NEG, respectively $(p<0.001$, $t$-test). The cut-off value of the correction angle per number of unfixed segments was $4^{\circ}$, determined using the ROC curve (specificity $=0.871$, sensitivity $=0.857$, area under the curve=0.894) (Fig. 2).

The change in the $\mathrm{O}-\mathrm{C} 7$ angle was $10.9^{\circ} \pm 4.5^{\circ}$ and $-0.8^{\circ} \pm 2.4^{\circ}$ in the FEG and NEG, respectively $(p=0.030, t$ -

Table 2. Background data before surgery in the non-extension and fusion extension groups

\begin{tabular}{|c|c|c|c|}
\hline Factors & Non-extension group ( $\mathrm{n}=26$ ) & Fusion extension group $(\mathrm{n}=9)$ & $p$-value \\
\hline Age (yr) & $61.5 \pm 8.7$ & $60.3 \pm 7.6$ & 0.647 (t-test) \\
\hline Sex (male:female) & $4: 22$ & $3: 6$ & 0.340 (Fisher exact test) \\
\hline RA type ${ }^{\text {a) }}$ & & & 0.769 (Fisher exact test) \\
\hline LES & 6 & 2 & \\
\hline MES & 12 & 6 & \\
\hline MUD & 1 & 0 & \\
\hline Unknown & 7 & 1 & \\
\hline Ranawat classification of neurological deficit & & & 0.494 (Fisher exact test) \\
\hline I & 0 & 0 & \\
\hline$\|$ & 7 & 4 & \\
\hline Illa & 12 & 3 & \\
\hline Illb & 3 & 2 & \\
\hline Unknown & 4 & 0 & \\
\hline Ranawat value (mm) & $9.6 \pm 1.1$ & $8.8 \pm 1.4$ & 0.664 (t-test) \\
\hline Space available for the spinal cord (mm) & $13.9 \pm 1.1$ & $16.8 \pm 1.4$ & 0.117 (t-test) \\
\hline Atlanto-dental interval (mm) & $8.1 \pm 0.8$ & $5.7 \pm 1.1$ & 0.081 (t-test) \\
\hline O-CLFV angleb) $\left(^{\circ}\right)$ & $16.6 \pm 2.8$ & $23.0 \pm 5.5$ & 0.312 (t-test) \\
\hline O-C7 angle $\left({ }^{\circ}\right)$ & $34.7 \pm 2.8$ & $41.9 \pm 5.3$ & 0.246 (t-test) \\
\hline
\end{tabular}

Values are presented as mean \pm standard deviation or number.

RA, rheumatoid arthritis; 0-C, occipitocervical.

${ }^{\text {al }}$ Type of RA: LES, the subset with least erosive disease; MES, the subset with more erosive disease; MUD, the subset with mutilating disease. ${ }^{\text {b) } O c-~}$ cipito to cervical lower fixed vertebra angle. 
test), and the change in the $\mathrm{O}-\mathrm{C} 7$ angle per number of unfixed segment was $4.7^{\circ} \pm 1.4^{\circ}$ and $0.4^{\circ} \pm 0.7^{\circ}$ in the FEG and NEG, respectively ( $p=0.009, t$-test). However, Ranawat value, atlanto-dental interval, space available for the spinal cord, and their translational change did not show a significant difference (Table 3 ).

\section{Change in the correction and compensation angles after occipitocervical reconstruction}

There was a mild correlation between the correction and compensation angles: $Y=-0.35 X-1.55, R=-0.451$ ( $Y$ : com-

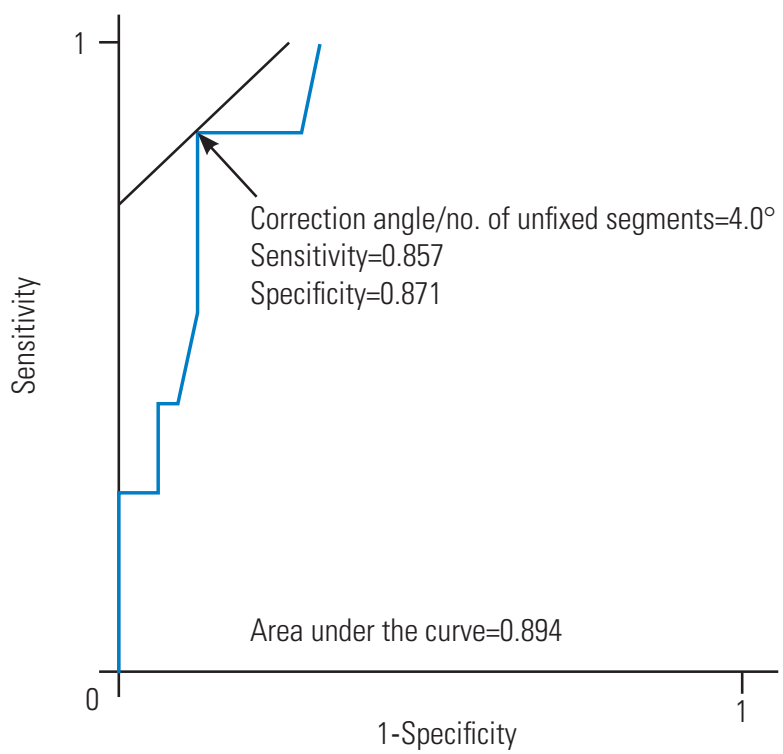

Fig. 2. Relationship between the correction and compensation angles. The graph shows the relationship between the correction and compensation angles without fusion extension reconstruction. We found a mild co-relation between the correction and compensation angles: $Y=-0.35 X-1.6, R=-0.451$ ( $Y$ : compensation angle, $X$ : correction angle). pensation angle, $X$ : correction angle) (Fig. 3).

\section{Discussion}

In the present study, the risk factors for FE reconstructions included $\mathrm{O}$-under $\mathrm{C} 2$ reconstruction, overcorrection against the residual unfixed mobile segments $\left(>4^{\circ}\right.$ per number of residual cervical unfixed segments), and change in the O-C7 angle. Basilar invagination, forward subluxation of the atlas, and these changes did not show risk factors for the late occurrence of subluxation or fracture at the end of the fixed vertebra requiring FE reconstruction.

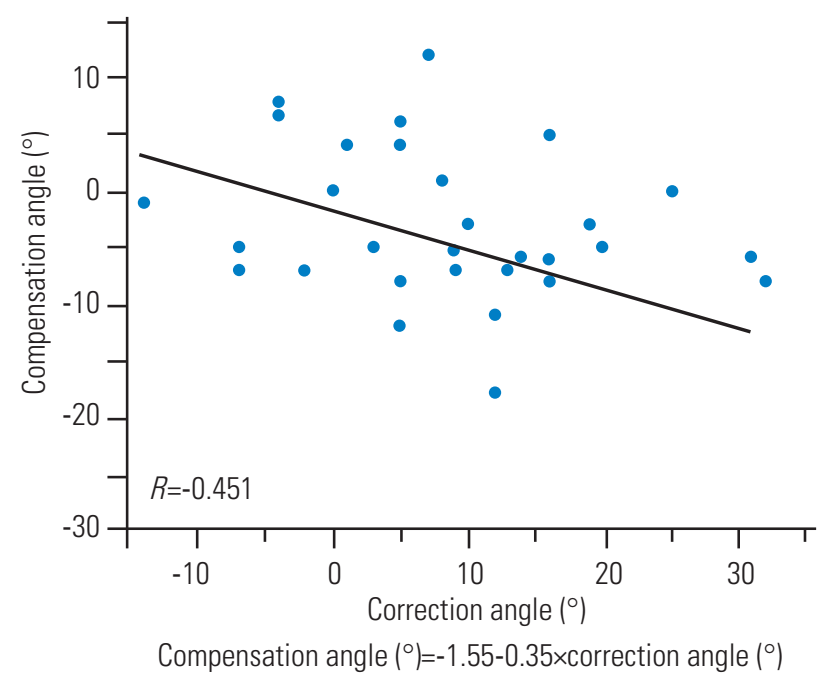

Fig. 3. The cut-off value of the correction angle per number of unfixed segments for the risk of late subaxial lesion. The graph showed the receiver operating curve of correction angle for the risk of late subaxial lesion. The cut-off value determined using the Youden Index was $4^{\circ}$ per the number of unfixed segments (area under the curve $=0.894$, sensitivity $=0.857$, specificity $=0.871$ ).

Table 3. Change of parameters at occipitocervical reconstruction in the non-extension and fusion extension groups

\begin{tabular}{|c|c|c|c|}
\hline Measurements & Non-extension group (n=26) & Fusion extension group ( $n=9)$ & $p$-value \\
\hline Change of Ranawat value & $4.4 \pm 0.9$ & $2.8 \pm 1.1$ & 0.250 \\
\hline Change of atlanto-dental interval & $-3.0 \pm 0.8$ & $-2.3 \pm 1.0$ & 0.609 \\
\hline Change of the space available for the spinal cord & $3.3 \pm 1.0$ & $1.9 \pm 1.3$ & 0.391 \\
\hline Correction angle $\left({ }^{\circ}\right)$ & $3.4 \pm 2.2$ & $9.9 \pm 4.7$ & 0.217 \\
\hline Change of $\mathrm{O}-\mathrm{C} 7$ angle $\left(^{\circ}\right)$ & $-0.8 \pm 2.4$ & $10.9 \pm 4.5$ & $0.030^{*}$ \\
\hline Correction angle/no. of un-fixed segment $\left({ }^{\circ}\right)$ & $0.8 \pm 1.0$ & $9.6 \pm 2.0$ & $0.001^{* *}$ \\
\hline Change of $0-\mathrm{C} 7$ angle/no. of un-fixed segment $\left({ }^{\circ}\right)$ & $0.4 \pm 0.7$ & $4.7 \pm 1.4$ & $0.009 * *$ \\
\hline
\end{tabular}

Values are presented as mean \pm standard deviation or number.

$0-C$, occipitocervical.

${ }^{*} p<0.05 .{ }^{* *} p<0.01$. 
O-under C2 correction, longer corrected fusion with larger mechanical stress for adjacent segment by longer fusion, fell into FE reconstruction more easily than O-C2 reconstruction. Although the correction angle was not significantly different between the FEG and the NEG, the $\mathrm{O}-\mathrm{C} 7$ angle changed after $\mathrm{O}-\mathrm{C}$ reconstruction in the FEG, which was different from that in the NEG. Based on this, the group that required $\mathrm{FE}$ reconstructions may have a lower ability to compensate the correction angle. The correction angle per number of unfixed mobile segments was introduced to consider the permissive correction angle for the secured mobile segments. The cut-off value was $4^{\circ}$, the limit of the kyphotic compensation angle multiplied by the number of unfixed segments. The ROC curve regarding it for the risk of FE reconstruction showed that it was an independent parameter for the risk of FE reconstruction (area under the curve was 0.894).

Reconstruction to gain lordosis at the $\mathrm{O}-\mathrm{C}$ junction would affect to change kyphotic at unfixed segments. A mild negative co-relationship between the correction and compensation angles to balance the cervical vertebra in the NEG and the correction angle at O-CLFV was almost completely compensated by the unfixed mobile segments at the CLFV-C7 in the NEG. Unfixed segments would compensate the correction angle to balance the cervical spine, resulting from the immovable O-C7 angle. Several studies have reported that the subaxial alignment changes to compensate the change in the atlanto-axial angle in atlanto-axial fusion [14-16] and the O-C2 angle in O-C2 fusion without a change in the $\mathrm{O}-\mathrm{C} 7$ angle $[17,18]$. Ishii et al. [16] showed that the average postoperative atlantoaxial angle in patients with late subaxial lesions was significantly larger than the average preoperative atlanto-axial angle in patients with late subaxial lesion. Furthermore, postoperative C2-7 angle in patients with late subaxial lesion was significantly smaller than that in patients without subaxial lesion [16]. Considering this, compensation in the un-instrumented mobile segments reached its limit in the FEG with a change in the $\mathrm{O}-\mathrm{C} 7$ angle after $\mathrm{O}-\mathrm{C}$ reconstruction.

Compensation in unfixed mobile segments was not accomplished against the correction angle in the FEG. The correction angle in the FEG was too large to compensate in the unfixed segments, resulting in a change in the O-C7 angle. A change in the $\mathrm{O}-\mathrm{C} 7$ angle leads to a change in the face angle. The patients with bone and joint fragility from RA would recover their O-C7 angle with lack of kyphotic flexibility at unfixed segments, attempting to drive the same position of their face after reconstruction as before reconstruction. Thereafter, late subaxial lesions as fractures or subluxation would occur to increase the kyphosis beyond the flexibility of the CLFV-C7 angle. Even if a reliable anchor is obtained in $\mathrm{O}-\mathrm{C}$ reconstruction, the correction angle should be conducted under the limit of the kyphotic compensation angle in CLFV-C7 segments, $>4^{\circ}$ per number of residual cervical unfixed segments. However, if the correction angle will be needed beyond the flexibility of unfixed segments, or if cases requiring the correction of $\mathrm{O}-\mathrm{C} 7$ angle as the cases needed the change of face angle like chin on chest position, occipito-cervico-thoracic fusion should be considered.

In the present study, translational value, such as Ranawat value, did not indicate the cause of FE reconstruction. Translational reduction at the atlanto-axial subluxation would be safer than the larger correction of the kyphotic deformation at the $\mathrm{O}-\mathrm{C}$ lesion. When an indirect decompression technique for $\mathrm{O}-\mathrm{C}$ reconstruction is performed, reduction of the atlanto-dental interval by anterior shift of axis should be performed before the correction of angulation, particularly in $\mathrm{O}$-under $\mathrm{C} 2$ reconstruction.

Our study has certain limitations that should be addressed. First, the patients included in this study did not show all the conditions needed for fusion extension reconstruction. Some patients may not undergo FE reconstruction owing to their general condition. Considering this, our study showed the ratio required for FE reconstruction in each range of the fusion segment. Second, this study targeted patients without a tight control with biological drugs; this might have affected the natural history of the facet joint by strong suppression of arthritis. However, even in patients under tight control with biological drugs, pre-existing joint destruction will progress [19]. In patients who need O-C reconstruction even under tight control with biological drugs, overcorrection should be limited. Further studies are required to confirm this finding. Third, the sample size of the control group was relatively small for this study population because of the low follow-up rate over 5 years.

\section{Conclusions}

Late subaxial lesions are known to develop occasionally following O-C reconstruction in patients with RA. The risk factors for late subaxial lesions included correction to 
subaxial segments, larger correction against mobile segments ( $4^{\circ}$ per number of unfixed segments), and change of the occipito-C7 angle after reconstruction. The correction angle should be limited, considering the cervical unfixed segments.

\section{Conflict of Interest}

No potential conflict of interest relevant to this article was reported.

\section{Acknowledgments}

We express sincere gratitude to our colleagues, nurses, physical therapists, X-ray technicians, pharmacists, and clerks at Hokkaido University Hospital, who provided care and support to the patients. The authors would like to thank Wolters Kluwer Author Services for the English language review.

\section{Author Contributions}

Akira Iwata conducted analysis and interpretation of data. Kuniyoshi Abumi was the main surgeon in this study, and contributed in acquisition of data. Masahiko Takahata supported the analysis and interpretation of data. Hideki Sudo, Katsuhisa Yamada, and Tsutomu Endo provided general support. Norimasa Iwasaki is the chair of the department who provided general support.

\section{References}

1. Wolfs JF, Kloppenburg M, Fehlings MG, van Tulder MW, Boers M, Peul WC. Neurologic outcome of surgical and conservative treatment of rheumatoid cervical spine subluxation: a systematic review. Arthritis Rheum 2009;61:1743-52.

2. Delamarter RB, Bohlman HH. Postmortem osseous and neuropathologic analysis of the rheumatoid cervical spine. Spine (Phila Pa 1976) 1994;19:2267-74.

3. Mikulowski P, Wollheim FA, Rotmil P, Olsen I. Sudden death in rheumatoid arthritis with atlanto-axial dislocation. Acta Med Scand 1975;198:445-51.

4. Smith HP, Challa VR, Alexander E Jr. Odontoid compression of the brain stem in a patient with rheumatoid arthritis. Case report. J Neurosurg 1980;53:8415 .
5. Monsey RD. Rheumatoid arthritis of the cervical spine. J Am Acad Orthop Surg 1997;5:240-8.

6. Eyres KS, Gray DH, Robertson P. Posterior surgical treatment for the rheumatoid cervical spine. Br J Rheumatol 1998;37:756-9.

7. Paus AC, Steen H, Roislien J, Mowinckel P, Teigland J. High mortality rate in rheumatoid arthritis with subluxation of the cervical spine: a cohort study of operated and nonoperated patients. Spine (Phila Pa 1976) 2008;33:2278-83.

8. Shen FH, Samartzis D, Jenis LG, An HS. Rheumatoid arthritis: evaluation and surgical management of the cervical spine. Spine J 2004;4:689-700.

9. Abumi K, Takada T, Shono Y, Kaneda K, Fujiya M. Posterior occipitocervical reconstruction using cervical pedicle screws and plate-rod systems. Spine (Phila Pa 1976) 1999;24:1425-34.

10. Wakao N, Takeuchi M, Nishimura M, et al. Vertebral artery variations and osseous anomaly at the C1-2 level diagnosed by 3D CT angiography in normal subjects. Neuroradiology 2014;56:843-9.

11. Miyata $\mathrm{M}, \mathrm{Neo} \mathrm{M}$, Ito $\mathrm{H}$, et al. Is rheumatoid arthritis a risk factor for a high-riding vertebral artery? Spine (Phila Pa 1976) 2008;33:2007-11.

12. Chung SS, Lee CS, Chung HW, Kang CS. CT analysis of the axis for transarticular screw fixation of rheumatoid atlantoaxial instability. Skeletal Radiol 2006;35:679-83.

13. Miyata $M$, Neo M, Fujibayashi S, Ito $H$, Takemoto $M$, Nakamura T. O-C2 angle as a predictor of dyspnea and/or dysphagia after occipitocervical fusion. Spine (Phila Pa 1976) 2009;34:184-8.

14. Yoshimoto $\mathrm{H}$, Ito $\mathrm{M}$, Abumi $\mathrm{K}$, et al. A retrospective radiographic analysis of subaxial sagittal alignment after posterior C1-C2 fusion. Spine (Phila Pa 1976) 2004;29:175-81.

15. Mukai Y, Hosono N, Sakaura H, et al. Sagittal alignment of the subaxial cervical spine after C1-C2 transarticular screw fixation in rheumatoid arthritis. J Spinal Disord Tech 2007;20:436-41.

16. Ishii K, Matsumoto M, Takahashi Y, et al. Risk factors for development of subaxial subluxations following atlantoaxial arthrodesis for atlantoaxial subluxations in rheumatoid arthritis. Spine (Phila Pa 1976) 2010;35:1551-5.

17. Matsubayashi Y, Shimizu T, Chikuda H, Takeshita K, Oshima Y, Tanaka S. Correlations of cervical sagittal 
alignment before and after occipitocervical fusion. Global Spine J 2016;6:362-9.

18. Nunez-Pereira S, Hitzl W, Bullmann V, Meier O, Koller H. Sagittal balance of the cervical spine: an analysis of occipitocervical and spinopelvic interdependence, with C-7 slope as a marker of cervical and spinopelvic alignment. J Neurosurg Spine
2015;23:16-23.

19. Kaito T, Ohshima S, Fujiwara H, Makino T, Yonenobu K. Predictors for the progression of cervical lesion in rheumatoid arthritis under the treatment of biological agents. Spine (Phila Pa 1976) 2013;38:225863. 